\title{
Three-point Green functions in the odd sector of QCD
}

\author{
T. Kadavý1, ${ }^{1,}, K . K^{\prime} a m p f^{1}$, and J. Novotný ${ }^{1}$ \\ ${ }^{1}$ Charles University, Faculty of Mathematics and Physics, Prague, Czech Republic
}

\begin{abstract}
A review of familiar results of the three-point Green functions of currents in the odd-intrinsic parity sector of QCD is presented. Such Green functions include very well-known examples of $V V P, V A S$ or $A A P$ correlators. We also shortly present some of the new results for $V V A$ and $A A A$ Green functions with a discussion of their high-energy behaviour and its relation to the QCD condensates.
\end{abstract}

\section{Introduction}

The Green functions (also called as correlators) are defined as the vacuum expectation values of the time ordered products of the composite operators. In our case, such operators stand either for chiral vector and axial-vector currents $V^{\mu, a}=\bar{q} \gamma^{\mu} T^{a} q, A^{\mu, a}=\bar{q} \gamma^{\mu} \gamma_{5} T^{a} q$, or scalar and pseudoscalar densities $S^{a}=\bar{q} T^{a} q, P^{a}=i \bar{q} \gamma_{5} T^{a} q$, where $q$ stands for the triplet of light quarks $(q=u, d, s), T^{a}$ are the SU(3) generators $\left(T^{a}=\lambda^{a} / 2\right)$, and $a=1 \ldots 8$ are the group indices. There exist only five nontrivial threepoint Green functions in the odd-intrinsic parity sector of QCD: $V V P, V A S, A A P, V V A$ and $A A A$.

There are two regimes where the QCD dynamics of the current correlators is well understood. The first one is that of low external momenta where the dynamics is governed by the means of chiral perturbation theory $(\chi \mathrm{PT})$ and resonance chiral theory $(\mathrm{R} \chi \mathrm{T})$. The second regime corresponds to the high energies where the asymptotic freedom allows us to use the perturbative approach in terms of the strong coupling $\alpha_{s}$ and where the asymptotics of the correlators for large euclidean momenta is given by the operator product expansion (OPE).

A consequence of the OPE is a presence of the vacuum avarages of composite gauge-invariant local operators, made of quark and gluon fields. Only the spin-zero operators, usually called the QCD condensates, contribute to the vacuum expectation value. Such nontrivial condensates with dimension $D \leq 6$ are shown here:

$$
\begin{array}{lll}
O_{3}=\langle 0|\bar{q} q| 0\rangle, & O_{4}=\left\langle 0\left|G_{\mu \nu} G^{\mu \nu}\right| 0\right\rangle, & O_{5}=\left\langle 0\left|\bar{q} \sigma_{\mu \nu} G^{\mu v} q\right| 0\right\rangle, \\
O_{6}^{q}=\langle 0|(\bar{q} \Gamma q)(\bar{q} \Gamma q)| 0\rangle, & O_{6}^{G}=\left\langle 0\left|G_{\mu \nu}^{a} G_{\sigma}^{\nu, b} G^{\sigma \mu, c}\right| 0\right\rangle f_{a b c}, &
\end{array}
$$

where $\Gamma$ stands for any combination of the $4 \times 4$ matrices $\left\{1, \gamma_{5}, \gamma^{\mu}, \gamma_{5} \gamma^{\mu}, \sigma^{\mu \nu}\right\}$ with $3 \times 3$ matrices $\left\{1, T^{a}\right\}$. In the following sections we will see that such QCD condensates play an important role in an evaluation of the OPE of the corresponding Green functions.

\footnotetext{
^e-mail: kadavy@ipnp.troja.mff.cuni.cz
} 


\section{Familiar results}

Familiar Green functions that have been already studied extensively within the odd sector of QCD include $V V P, V A S$ and $A A P$ correlators. Since their tensor structure is similar, let us take the $V V P$ correlator as an example and write down its structure in the form ${ }^{1}$

$$
\left(\Pi_{V V P}(p, q ; r)\right)_{\mu \nu}^{a b c}=\Pi_{V V P}\left(p^{2}, q^{2} ; r^{2}\right) d^{a b c} \varepsilon_{\mu v(p)(q)} .
$$

The high-energy behaviour within the OPE framework can be written down as follows. If we consider all individual external momenta of all the three currents or densities to be large, it is easy to realize that the leading order contribution, where only the quark condensate contributes, corresponds to two contractions of the quark fields, contained in the respective currents and densities. Such contribution can be illustrated as

$$
\langle 0|\rightarrow \bigotimes \rightarrow-\otimes \rightarrow-\otimes \rightarrow-| 0\rangle \neq 0,
$$

where the crossed circle stands for an insertion either of the currents or the densities. The explicit calculation leads to the result [1]

$$
\Pi_{V V P}\left((\lambda p)^{2},(\lambda q)^{2} ;(\lambda r)^{2}\right)=\frac{B_{0} F^{2}}{2 \lambda^{4}} \frac{p^{2}+q^{2}+r^{2}}{p^{2} q^{2} r^{2}}+O\left(\frac{1}{\lambda^{6}}\right) .
$$

Calculation of $V V P$ (and also $V A S$ ) correlator in the odd sector up to $O\left(p^{6}\right)$ was provided in [2], together with a study of the OPE and other phenomenological applications.

\section{OPE for $V V A$ Green function with two large momenta}

Before we advance, let us make a use of the known result of the OPE for the $V V A$ correlator, where only two external momenta are considered to be large. The Ward identities restrict the general decomposition of the tensor part of the $V V A$ into four terms,

$$
\left(\Pi_{V V A}(p, q ; r)\right)_{\mu \nu \rho}^{a b c} \equiv d^{a b c}\left(w_{L} \varepsilon_{\mu \nu(p)(q)} r_{\rho}+w_{T}^{(1)} \Pi_{\mu \nu \rho}^{(1)}+w_{T}^{(2)} \Pi_{\mu \nu \rho}^{(2)}+w_{T}^{(3)} \Pi_{\mu \nu \rho}^{(3)}\right),
$$

with the longitudinal part $w_{L}$ fixed by the anomaly term and the transversal part defined in [3]. The formfactors $w_{L}$ and $w_{T}^{(i)}, i=1,2,3$, were obtained in the case of the odd-intrinsic parity sector up to $O\left(p^{6}\right)$ in [6]. Then, one can introduce the phenomenologically important formactor [3]

$$
w_{T}\left(Q^{2}\right)=-16 \pi^{2}\left[w_{T}^{(1)}\left(-Q^{2}, 0,-Q^{2}\right)+w_{T}^{(3)}\left(-Q^{2}, 0,-Q^{2}\right)\right] .
$$

It turns out that the result for (6) up to $O\left(1 / Q^{8}\right)$ can be also obtained from soft wall AdS/QCD and the OPE framework for two large external momenta, from which the outcome reads [4], [5]

$$
w_{T}\left(Q^{2}\right)=\frac{N_{c}}{Q^{2}}+\frac{128 \pi^{3} \alpha_{s} \chi\langle\bar{q} q\rangle^{2}}{9 Q^{6}}+O\left(\frac{1}{Q^{8}}\right) .
$$

Our result [6] for (6) can be expanded into a series in terms of $Q^{2}$ up to $O\left(1 / Q^{8}\right)$ and compared with individual terms in (7). This procedure leads to several constraints for the coupling constants and also for the prediction for the deviation $\delta_{\mathrm{BL}}$, that propagates itself in the $\kappa_{3}^{P V}$ constraint, if we take the Brodsky-Lepage behaviour of the transition form factor $\mathcal{F}_{\pi^{0} \gamma \gamma}$ into account [2], [7], [8]. Our calculations predict $\delta_{\mathrm{BL}}=-1.342$. However, such value is not in an agreement with the experimental data and one, therefore, has to rule out the OPE with two large momenta as a relevant OPE in the case of the $V V A$ Green function (see [6] for a detailed discussion).

\footnotetext{
${ }^{1}$ In the following text we consider all momenta $p, q, r$ as ingoing to the vertices, i.e. the law of momentum conservation is $p+q+r=0$. Also, a short-hand notation for the contractions of Levi-Civita tensor with the components of momenta is defined as $\varepsilon_{\mu \nu \alpha(p)} \equiv \varepsilon_{\mu \nu \alpha \beta} p^{\beta}, \varepsilon_{\mu v(p)(q)} \equiv \varepsilon_{\mu \nu \alpha \beta} p^{\alpha} q^{\beta}$.
} 


\section{OPE for $V V A$ and $A A A$ Green functions with three large momenta}

Following the discussion above, one can try to calculate the OPE for $V V A$ and also for $A A A$ correlators and consider all three momenta to be large. The motivation is to find out if the results will become consistent with the experimental data. However, such an approach is not trivial in this case. None of these correlators have the leading order contribution to the OPE, i.e. the contributions of the topology (3) identically vanishes. Therefore, one is required to include the contributions to higher orders by means of diagrams with gluons coupled to the quark fields. Such diagrams naturally lead to contributions of higher QCD condensates. The scheme below shows the contributions of the quark condensate $(D=3)$, the gluon condensate $(D=4)$, the quark-gluon condensate $(D=5)$, the fourquark condensate and the three-gluon condensate $(D=6)$. This study is currently in progress [6].

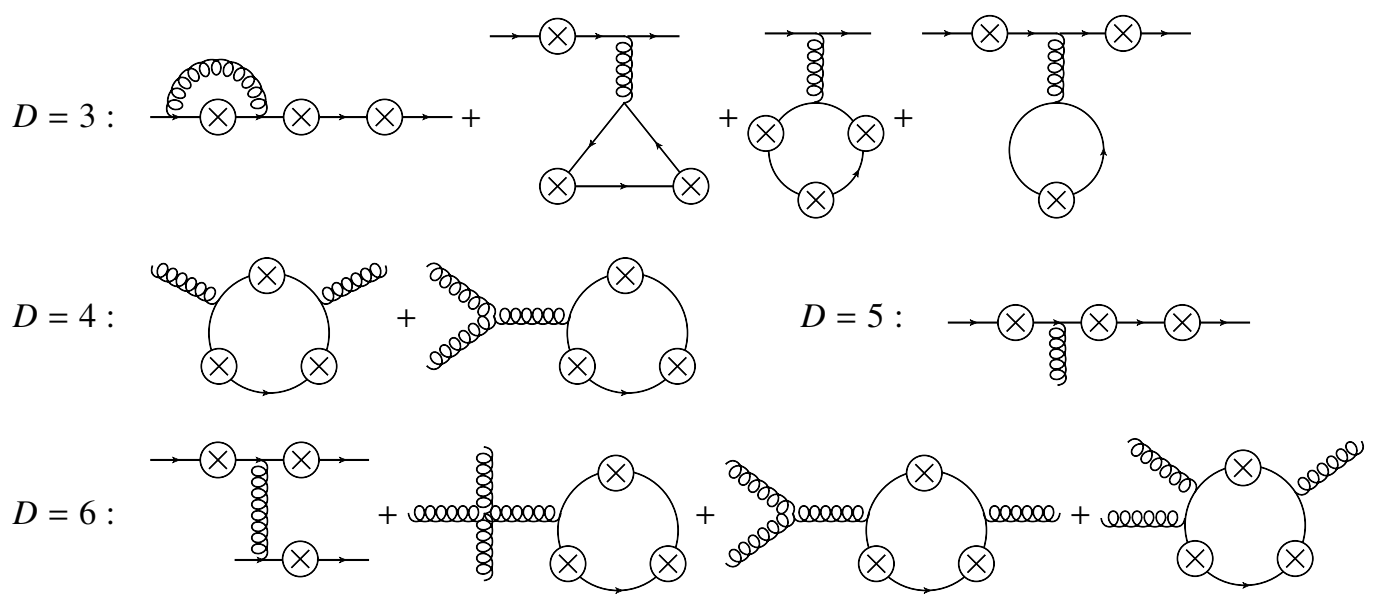

\section{Conclusion}

In this proceeding contribution, we summarized the known results for the high-energy behaviour of Green functions within the OPE framework, relevant in the odd-intrinsic parity sector. We also presented our approach in order to evaluate the OPE for the $V V A$ and $A A A$ correlators, where all momenta are large.

Acknowledgments. TK thanks the organizers for a pleasant conference. The support by the Charles University (project GA UK no. 700214) and by The Czech Science Foundation (project GA CR no. 15-18080S) is gratefully acknowledged.

\section{References}

[1] B. Moussallam, Phys. Rev. D 51, 4939-4949 (1995)

[2] K. Kampf et al., Phys. Rev. D 84, 014036 (2011)

[3] M. Knecht et al., JHEP 0403, 035 (2004)

[4] P. Colangelo et al., Phys. Rev. D 85, 035013 (2012)

[5] J. J. Sanz-Cillero, PoS QNP 2012, 118 (2012)

[6] T. Kadavý et al. (in preparation)

[7] G. P. Lepage et al., Phys. Rev. D 22, 2157 (1980)

[8] S. J. Brodsky et al., Phys. Rev. D 24, 1808 (1981) 